\title{
New fixed point results in quasi-metric spaces and applications in fractals theory
}

\author{
Nicolae Adrian Secelean ${ }^{1 *}$ (D, Sunil Mathew ${ }^{2}$ and Dariusz Wardowski ${ }^{3}$
}

\section{*Correspondence:}

nicolae.secelean@ulbsibiu.ro

1 Department of Mathematics and

Computer Science, Lucian Blaga

University of Sibiu, Sibiu, Romania

Full list of author information is

available at the end of the article

\section{Springer}

\begin{abstract}
In this paper, we prove some fixed point theorems for $\psi F$-contractions in the framework of quasi-metric spaces generalizing and improving several similar results in metric spaces. At the same time, we consider iterated function systems consisting of $\psi \mathrm{F}$-contractions on quasi-metric spaces, and we give some sufficient conditions for the existence and uniqueness of their attractor which is, generally, a fractal. Some illustrative examples are provided.
\end{abstract}

MSC: Primary 47H10; 28A80; secondary 47H09; 54E50; 54E55

Keywords: Quasi-metric space; Forward (backward) F-contraction; Picard operator; Iterated function system; Fractals

\section{Introduction}

In the literature known by the authors, the quasi-metric (asymmetric) space notion can be traced back to W.A. Wilson [1]. This is defined as metric space $(X, \mathrm{~d})$ but without the symmetry requirement for $\mathrm{d}$.

Quasi-metric spaces have numerous recent applications both in pure and applied mathematics, for example, in the questions of existence and uniqueness of Hamilton-Jacobi equations [2], in rate-independent models for plasticity [3], shape-memory alloys [4], models for material failure [5], automated taxonomy construction [6], and so on.

In quasi-metric spaces some concepts, such as convergence, continuity, compactness, and completeness, are different from those in metric case. There are two notions for each of them, namely forward and backward ones, since we have two topologies which are the forward topology and the backward topology in quasi-metric spaces (see [7]). In the last decades many authors studied these notions and properties in the settings of quasi-metric spaces (see, e.g., [8-16] and the references therein).

Starting from the Banach contraction principle, which is a pivotal result of analysis, many authors have provided several extensions of this result by considering more general spaces and various types of contractions. Wardowski defined in [17] $F$-contraction as a mapping $T$ on a metric space $(X, \mathrm{~d})$ into itself such that $\tau+F(\mathrm{~d}(T x, T y)) \leq F(\mathrm{~d}(x, y))$ for all $x, y \in X$ with $T x \neq T y$, where $\tau>0$ and $F:(0, \infty) \rightarrow \mathbb{R}$ satisfies the following axioms: (F1) $F$ is increasing, (F2) $F(t) \rightarrow-\infty$ if and only if $t \searrow 0$, and (F3) $\lim _{t \rightarrow 0} t^{\lambda} F(t)=0$ for some $\lambda \in(0,1)$. For such contractions, he obtained a classical fixed point result concerning the existence and uniqueness of fixed point which is the limit of the Picard iterations. Putting

(c) The Author(s) 2019. This article is distributed under the terms of the Creative Commons Attribution 4.0 International License (http://creativecommons.org/licenses/by/4.0/), which permits unrestricted use, distribution, and reproduction in any medium, provided you give appropriate credit to the original author(s) and the source, provide a link to the Creative Commons license, and indicate if changes were made. 
certain concrete forms of $F$, Wardowski obtained other known types of contractions, including Banach contraction for $F(t)=\ln t$, and proved that $F$-contractions are really their generalizations.

Next, some other generalizations of $F$-contractions have been studied by many authors. In this respect, Secelean and Wardowski [18] introduced $\psi F$-contractions as being selfmappings $T$ satisfying the inequality $F(\mathrm{~d}(T x, T y)) \leq \psi(F(\mathrm{~d}(x, y)))$ for all $x, y \in X, T x \neq T y$, where $\psi:(-\infty, \mu) \rightarrow(-\infty, \mu)$ is increasing and $\psi^{n}(t) \rightarrow-\infty$ for all $t \in(-\infty, \mu), \mu=\sup F$. In the above mentioned paper, some fixed point results are given even if $F$ does not satisfy all conditions (F1)-(F3). Very recently, Nazama, Arshada, and Postolache [19] gave some interesting results concerning coincidence and common fixed points for four mappings satisfying certain $F$-contraction type conditions. For other generalizations and applications of $F$-contractions, one can also see [20] and [21].

Our first purpose in the present paper is to extend and improve the theory of $\psi F$ contractions in the settings of quasi-metric spaces (Sect. 3.1). Furthermore, we prove some fixed point results (Theorems 3.1, 3.2, 3.3 and their corollaries) which generalize and improve those from $[17,18,22]$ and from many others in two directions: the first one is the space where the contraction is defined, and in the second one the function $F$ must satisfy only condition (F1).

In order to do this, in Sect. 2 we define the quasi-metric space and next we state the main concepts and properties in this space that will be used in the sequel, such as forward topology, forward convergence, forward compactness, forward completeness, forward boundedness, and so on, and analogs for backward.

The second goal of this paper is to survey, as an application, some fractals generated in quasi-metric spaces. As it is well known, fractals theory is one of the modern and dynamic fields in mathematics with a spectacular development in recent decades due to their applicability in various areas of science and technology.

In his famous paper [23] Hutchinson called Iterated Function Systems (for short IFS) a finite family of Banach contractions $\left(\omega_{k}\right)_{k=1}^{N}$ on a metric space $X$ and defined a set value function $\mathcal{S}$ on the class of all non-empty compact subsets of $X$ into itself by $\mathcal{S}(B)=\bigcup_{k=1}^{N} \omega_{k}(B)$; he also proved that, if the metric space is complete, then $\mathcal{S}$ has a unique set fixed point $A$. The set $A$ is generally a fractal set called the attractor of the respective IFS. The IFSs are the main generators of fractals. There is a current effort to extend Hutchinson's classical framework for fractals to more general spaces and infinite IFSs or, more generally, to multifunction systems.

For our purpose, we need some preliminary results concerning the Hausdorff-Pompeiu metric in the framework of quasi-metric space (Sect. 3.2). Some sufficient completeness conditions for the spaces of fractals are provided in Theorem 3.4.

Existence and uniqueness of the attractors of IFSs composed of $\psi F$-contractions are given in Sect. 4 where Theorems 4.1, 4.2, 4.3, and 4.4 generalize and improve the analogous results in metric spaces.

Some illustrative examples are given.

\section{Preliminaries}

Throughout this paper the symbols $\mathbb{R}, \mathbb{R}_{+}$, and $\mathbb{N}$ denote the sets of all real numbers, positive real numbers, and positive integers, respectively. We also write $\overline{\mathbb{R}}_{+}=\mathbb{R}_{+} \cup\{\infty\}$.

If $v, \lambda \in \overline{\mathbb{R}}_{+}$, by " $v \geq \lambda$ " we understand $v \geq \lambda$ if $\lambda \in \mathbb{R}_{+}$and $v=\infty$ otherwise. 
For a mapping $T: E \rightarrow E$, by $T^{n}$ we denote the $n$-times composition of $T, T^{n+1}=T \circ T^{n}$, $n \in \mathbb{N}$.

\subsection{Quasi-metric spaces}

We consider $X \neq \emptyset$.

Definition 2.1 Let $\mathrm{d}$ be a non-negative real-valued function on the product $X \times X$ and consider the following axioms:

(M1) $\mathrm{d}(x, x)=0, \forall x \in X$;

(M2) $\mathrm{d}(x, y)=0 \Rightarrow x=y$;

(M3) $\mathrm{d}(x, y)=\mathrm{d}(y, x), \forall x, y \in X$;

(M4) $\mathrm{d}(x, z) \leq \mathrm{d}(x, y)+\mathrm{d}(y, z), \forall x, y, z \in X$.

We call d pseudo-metric if it satisfies (M1), (M3), (M4); quasi-metric or asymmetric metric if it satisfies (M1), (M2), (M4); quasi-pseudo-metric if it satisfies (M1) and (M4); and, respectively, metric if all axioms (M1)-(M4) are satisfied.

Accordingly, the pair $(X, \mathrm{~d})$ is called a pseudo-metric, quasi-metric (asymmetric metric), quasi-pseudo-metric, and metric space, respectively.

Throughout the paper we denote by $(X, \mathrm{~d})$ a quasi-metric space unless otherwise stated.

Definition 2.2 The forward topology $\mathcal{T}_{\mathrm{f}}$ induced by $\mathrm{d}$ is the topology generated by the forward open balls $\mathrm{B}_{\mathrm{f}}(x, \varepsilon)=\{y \in X ; \mathrm{d}(x, y)<\varepsilon\}$ for $x \in X, \varepsilon>0$.

Likewise, the backward topology $\mathcal{T}_{\mathrm{b}}$ induced by $\mathrm{d}$ is the topology generated by the backward open balls $\mathrm{B}_{\mathrm{b}}(y, \varepsilon)=\{x \in X ; \mathrm{d}(x, y)<\varepsilon\}$ for $y \in X, \varepsilon>0$.

A set $F \subset X$ is called $f$-closed (resp. $b$-closed) if $\complement F \in \mathcal{T}_{\mathrm{f}}$ (resp. $\complement F \in \mathcal{T}_{\mathrm{b}}$ ).

$\operatorname{Remark} 2.1$ If $(X, \mathrm{~d})$ is a quasi-metric space, then both $\mathcal{T}_{\mathrm{f}}$ and $\mathcal{T}_{\mathrm{b}}$ are T1-topologies; hence, in the topological spaces $\left(X, \mathcal{T}_{\mathrm{f}}\right)$ and $\left(X, \mathcal{T}_{\mathrm{b}}\right)$, the finite sets are closed.

In the following we present some usual examples of quasi-metric space.

Example 2.1 Let $\alpha>0$ and $\mathrm{d}: \mathbb{R} \times \mathbb{R} \rightarrow \mathbb{R}_{+} \cup\{0\}$ be defined by

$$
\mathrm{d}(x, y):= \begin{cases}y-x, & \text { if } y \geq x, \\ \alpha(x-y), & \text { if } y<x .\end{cases}
$$

Then $(\mathbb{R}, \mathrm{d})$ is a quasi-metric space and both $\mathcal{T}_{\mathrm{f}}$ and $\mathcal{T}_{\mathrm{b}}$ are the usual topology on $\mathbb{R}$.

More generally, if we consider an increasing function $f: \mathbb{R} \rightarrow \mathbb{R}$ and $\alpha>0$ and take

$$
\mathrm{d}(x, y):= \begin{cases}f(y)-f(x), & \text { if } y \geq x \\ \alpha(f(x)-f(y)), & \text { if } y<x\end{cases}
$$

we obtain also a quasi-metric on $\mathbb{R}$. If $f$ is continuous, both $\mathcal{T}_{\mathrm{f}}$ and $\mathcal{T}_{\mathrm{b}}$ are the usual topology on $\mathbb{R}$.

Example 2.2 The function $\mathrm{d}: \mathbb{R} \times \mathbb{R} \rightarrow \mathbb{R}_{+} \cup\{0\}$ defined by

$$
\mathrm{d}(x, y):= \begin{cases}e^{y}-e^{x}, & \text { if } y \geq x, \\ e^{-y}-e^{-x}, & \text { if } y<x\end{cases}
$$

is a quasi-metric. Both $\mathcal{T}_{\mathrm{f}}$ and $\mathcal{T}_{\mathrm{b}}$ are the usual topology on $\mathbb{R}$. 
Example 2.3 Let $(X, \mathrm{~d})$ be a quasi-metric space and $f: X \rightarrow X$ be a mapping. Then the function $\delta: X \times X \rightarrow \mathbb{R}_{+} \cup\{0\}$ defined by

$$
\delta(x, y):=\mathrm{d}(f(x), f(y)), \quad \forall x, y \in X
$$

is a quasi-pseudo-metric. Further, $\delta$ is a quasi-metric if and only if $f$ is injective.

Example 2.4 The function $\mathrm{d}: \mathbb{R} \times \mathbb{R} \rightarrow \mathbb{R}_{+} \cup\{0\}$ defined by

$$
\mathrm{d}(x, y):= \begin{cases}y-x, & \text { if } y \geq x \\ 1, & \text { if } y<x\end{cases}
$$

is a quasi-metric named Sorgenfrey quasi-metric. Here, $\mathcal{T}_{\mathrm{f}}$ is the lower limit topology on $\mathbb{R}$ and it is well known that $\mathcal{T}_{\mathrm{f}}$ is not metrizable (see, e.g., [24]). At the same time $\mathcal{T}_{\mathrm{b}}$ is the upper limit topology.

Definition 2.3 A sequence $\left(x_{n}\right)$ forward converges (shortly $f$-converges) to $x_{0} \in X$ (resp. backward converges (b-converges) to $x_{0} \in X$ ) if it converges with respect to the topology $\mathcal{T}_{\mathrm{f}}$ (resp. $\left.\mathcal{T}_{\mathrm{b}}\right)$. Accordingly, $\left(x_{n}\right)$ f-converges (resp. b-converges) to $x_{0}$ iff

$$
\mathrm{d}\left(x_{0}, x_{n}\right) \rightarrow 0, \quad \text { respectively } \mathrm{d}\left(x_{n}, x_{0}\right) \rightarrow 0 .
$$

For f-convergence and b-convergence, we shall use the notations: $x_{n} \stackrel{f}{\longrightarrow} x_{0}$ and $x_{n} \stackrel{b}{\longrightarrow}$ $x_{0}$, respectively.

Notice that the topology associated with a quasi-metric space is not generally Hausdorff (see, e.g., [7, Ex. 5.7]). A sufficient condition under which this property holds is given in the following proposition.

Proposition 2.1 If in the quasi-metric space $(X, \mathrm{~d}) f$-convergence implies $b$-convergence, then the topological space $\left(X, \mathcal{T}_{\mathrm{f}}\right)$ is Hausdorff.

Proof Let us consider $x \neq y \in X$. We claim that there is $N \in \mathbb{N}$ such that

$$
\mathrm{B}_{\mathrm{f}}\left(x, \frac{1}{N}\right) \cap \mathrm{B}_{\mathrm{f}}\left(y, \frac{1}{N}\right)=\emptyset .
$$

Indeed, on the contrary, for each $n \in \mathbb{N}$, one can find $z_{n} \in X$ such that $\mathrm{d}\left(x, z_{n}\right)<\frac{1}{n}$ and $\mathrm{d}\left(y, z_{n}\right)<\frac{1}{n}$. Hence $z_{n} \stackrel{f}{\longrightarrow} x, z_{n} \stackrel{f}{\longrightarrow} y$ and, by hypothesis, $z_{n} \stackrel{b}{\longrightarrow} y$. Consequently,

$$
0<\mathrm{d}(x, y) \leq \mathrm{d}\left(x, z_{n}\right)+\mathrm{d}\left(z_{n}, y\right) \underset{n}{\longrightarrow} 0,
$$

which is a contradiction.

Definition 2.4 We say that the sequence $\left(x_{n}\right) \subset X$ is forward Cauchy (resp. backward Cauchy) if, for each $\varepsilon>0$, there exists $N \in \mathbb{N}$ such that, for every $m \geq n \geq N$, one has

$$
\mathrm{d}\left(x_{n}, x_{m}\right)<\varepsilon, \quad\left(\text { resp. } \mathrm{d}\left(x_{m}, x_{n}\right)<\varepsilon\right) .
$$


The quasi-metric space $(X, \mathrm{~d})$ is forward complete (resp. backward complete) if every forward (resp. backward) Cauchy sequence is f-convergent (resp. b-convergent).

Definition 2.5 A set $A \subset X$ is forward bounded (f-bounded), respectively backward bounded (b-bounded), if there exists $x \in X$ such that $\sup _{y \in A} \mathrm{~d}(x, y)<\infty$, resp. there is $y \in A$ such that $\sup _{x \in A} \mathrm{~d}(x, y)<\infty$. $A$ is called bounded (fb-bounded) if it is both forward and backward bounded, i.e., $\operatorname{diam} A=\sup _{x, y \in A} \mathrm{~d}(x, y)<\infty$.

A is $f$-totally bounded if, for every $\varepsilon>0$, there are $x_{1}, x_{2}, \ldots, x_{n} \in A$ such that $A \subset$ $\bigcup_{k=1}^{n} \mathrm{~B}_{\mathrm{f}}\left(x_{k}, \varepsilon\right)$. Analogously we define $b$-totally bounded set.

It is easy to verify that a set $A \subset X$ is $\mathrm{f}$-bounded if and only if, for every $x \in X$, $\sup _{y \in A} \mathrm{~d}(x, y)<\infty$. An analogous property holds for a b-bounded set.

It is also obvious that every f-totally (b-totally) bounded set is f-bounded (b-bounded).

Definition 2.6 A compact set in the topological space $\left(X, \mathcal{T}_{\mathrm{f}}\right)$, respectively in $\left(X, \mathcal{T}_{\mathrm{b}}\right)$, is called $f$-compact, resp. b-compact. We say $K \subset X$ is $f$-sequentially (resp. $b$-sequentially) compact if every sequence has a forward (resp. backward) convergent subsequence with limit in $K$.

Theorem 2.1 ([11, Th. 4.8]) A quasi-metric space is f-compact (resp. b-compact) if and only if it is f-complete (resp. b-complete) andf-totally (resp. b-totally) bounded.

Proposition 2.2 ([14]) A sequentiallyf-compact quasi-pseudo-metric space X isf-compact if and only if $f$-closure of $\{x\}$ is $f$-compact for every $x \in X$. A similar assertion holds if we consider "backward" instead of "forward".

The proof of the following lemma is very similar to that in the metric case and hence it will be omitted.

Lemma 2.1 An f-compact (b-compact) set in a quasi-metric space is $f$-sequentially (bsequentially) compact.

By Lemma 2.1, Remark 2.1, and Proposition 2.2, we obtain the following obvious result.

Corollary 2.1 In a quasi-metric space a set is f-compact (resp. b-compact) if and only if it is $f$-sequentially (resp. $b$-sequentially) compact.

Proposition 2.3 If in the quasi-metric space $(X, \mathrm{~d}) f$-convergence implies $b$-convergence, every f-compact set is b-totally bounded, so it is b-bounded.

Proof Let $\emptyset \neq K \subset X$ be f-compact and assume by contradiction that there exists $\varepsilon>0$ such that $K$ cannot be covered by a finite number of backward open balls $\mathrm{B}_{\mathrm{b}}(y, \varepsilon), y \in K$. Choose $y_{1} \in K$. Since $K \not \subset \mathrm{B}_{\mathrm{b}}\left(y_{1}, \varepsilon\right)$, one can find $y_{2} \in K \backslash \mathrm{B}_{\mathrm{b}}\left(y_{1}, \varepsilon\right)$. Inductively, as $K \not \subset$ $\bigcup_{i=1}^{n} \mathrm{~B}_{\mathrm{b}}\left(y_{i}, \varepsilon\right)$, we can take $y_{n+1} \in K \backslash \bigcup_{i=1}^{n} \mathrm{~B}_{\mathrm{b}}\left(y_{i}, \varepsilon\right)$, and so on. In this way we obtain a sequence $\left(y_{n}\right) \subset K$ such that

$$
\mathrm{d}\left(y_{n+1}, y_{i}\right) \geq \varepsilon, \quad \forall n \in \mathbb{N}, \forall i \in\{1, \ldots, n\} .
$$


According to Lemma 2.1, there exists a subsequence $\left(y_{n_{k}}\right) \subset\left(y_{n}\right)$ such that $y_{n_{k}} \underset{k}{\stackrel{f}{\longrightarrow}} y \in K$. By hypothesis, one has also $y_{n_{k}} \stackrel{b}{\longrightarrow} y$. Hence, there exists $k_{0} \in \mathbb{N}$ such that $\mathrm{d}\left(y, y_{n_{k}}\right)<\frac{\varepsilon}{2}$ and $\mathrm{d}\left(y_{n_{k}}, y\right)<\frac{\varepsilon}{2}$ for every $k \geq k_{0}$. Consequently, using (2.1), we get

$$
\varepsilon \leq \mathrm{d}\left(y_{n_{k}+1}, y_{n_{k}}\right) \leq \mathrm{d}\left(y_{n_{k}+1}, y\right)+\mathrm{d}\left(y, y_{n_{k}}\right)<\varepsilon,
$$

which is a contradiction.

Definition 2.7 Let $(X, \mathrm{~d}),(Y, \rho)$ be two quasi-metric spaces. A mapping $f: X \rightarrow Y$ is said to be ff-continuous at $x \in X$ if and only if whenever $x_{n} \stackrel{f}{\longrightarrow} x$ in $(X, \mathrm{~d})$ one has $f\left(x_{n}\right) \stackrel{f}{\longrightarrow} f(x)$ in $(Y, \rho)$. If $Y=\mathbb{R}$ we say that $f$ is $f$-continuous at $x \in X$ if $\mathbb{R}$ is endowed with the Euclidean metric. We say that $f$ is $f f$-continuous ( $f$-continuous) if it is ff-continuous (f-continuous) at every $x \in X$. Analogous statements hold for the other continuity concepts: $f b$-continuous, bf-continuous, bb-continuous, b-continuous.

Proposition 2.4 If in the quasi-metric space $(X, \mathrm{~d}) f$-convergence implies $b$-convergence, then the mapping $(x, y) \mapsto \mathrm{d}(x, y)$ is f-continuous.

Proof Let us consider two sequences $\left(x_{n}\right),\left(y_{n}\right), x_{n} \stackrel{f}{\longrightarrow} x, y_{n} \stackrel{f}{\longrightarrow} y$, and $\varepsilon>0$. Then, by hypothesis, we have $x_{n} \stackrel{b}{\longrightarrow} x$ and $y_{n} \stackrel{b}{\longrightarrow} y$. Thus one can find $N \in \mathbb{N}$ such that

$$
n \geq N \quad \Rightarrow \quad \mathrm{d}\left(x, x_{n}\right)<\frac{\varepsilon}{2}, \quad \mathrm{~d}\left(y, y_{n}\right)<\frac{\varepsilon}{2}, \quad \mathrm{~d}\left(x_{n}, x\right)<\frac{\varepsilon}{2}, \quad \mathrm{~d}\left(y_{n}, y\right)<\frac{\varepsilon}{2} .
$$

Hence, for every $n \geq N$,

$$
\mathrm{d}\left(x_{n}, y_{n}\right)-\mathrm{d}(x, y) \leq \mathrm{d}\left(x_{n}, x\right)+\mathrm{d}\left(y, y_{n}\right)<\varepsilon
$$

and

$$
\mathrm{d}(x, y)-\mathrm{d}\left(x_{n}, y_{n}\right) \leq \mathrm{d}\left(x, x_{n}\right)+\mathrm{d}\left(y_{n}, y\right)<\varepsilon .
$$

Therefore

$$
\left|\mathrm{d}\left(x_{n}, y_{n}\right)-\mathrm{d}(x, y)\right|<\varepsilon, \quad \forall n \geq N .
$$

\section{The results}

\section{1 $\psi$ F-Contractions in quasi-metric spaces}

In this section we give some fixed point results in the setting of quasi-metric space which generalize and improve some ones proved in metric spaces.

According to Ćirić [25], we will adapt some concepts to quasi-metric settings.

Definition 3.1 A self-map $T$ on a quasi-metric space $(X, \mathrm{~d})$ is said to be $f b$-orbitally continuous at a point $x_{0} \in X$ if, for every increasing sequence $\left(n_{k}\right)_{k} \subset \mathbb{N}, T^{n_{k}} x_{0} \stackrel{f}{\longrightarrow} u \in X \mathrm{im}$ plies $T^{n_{k}+1} x_{0} \stackrel{b}{\longrightarrow} T u$. We say that $T$ is $f b$-orbitally continuous if it is fb-orbitally continuous at every $x \in X$. In a similar way we can define ff-orbital continuity, bf-orbital continuity, bb-orbital continuity, respectively. The space $X$ is $\mathrm{f}$ - $T$-orbitally complete if every $\mathrm{f}$-Cauchy sequence of the form $\left(T^{n_{k}} x\right)_{k} f$-converges in $X$. 
It is obvious that if $T$ is ff-continuous (resp. bb-continuous), then it is ff-orbitally (bborbitally) continuous, and if the space is $\mathrm{f}$-complete, then it is $\mathrm{f}$ - $T$-orbitally complete.

Lemma 3.1 ([26, L.3.2.a)]) Let $F: \mathbb{R}_{+} \rightarrow \mathbb{R}$ be an increasing map and $\left(t_{n}\right)_{n}$ be a sequence of positive real numbers such that $F\left(t_{n}\right) \underset{n}{\longrightarrow}-\infty$. Then $t_{n} \underset{n}{\longrightarrow} 0$.

In order to prove the next theorem, we need to adapt to our setting the following result given in [27, Prop. 3].

Proposition 3.1 Let $\left(x_{n}\right)$ be a sequence of elements from the quasi-metric space $(X, \mathrm{~d})$ and $\Delta$ be a subset of $\mathbb{R}_{+}$such that $\mathbb{R}_{+} \backslash \Delta$ is dense in $\mathbb{R}_{+}$. If $\mathrm{d}\left(x_{n}, x_{n+1}\right) \underset{n}{\longrightarrow} 0$ and $\left(x_{n}\right)$ is not $f$-Cauchy, then there exist $\varepsilon \in \mathbb{R}_{+} \backslash \Delta, n_{0} \in \mathbb{N}$, and sequences of positive integers $\left(m_{k}\right),\left(n_{k}\right)$ such that

(i) $\forall k \in \mathbb{N}, k \leq m_{k}<n_{k}, \mathrm{~d}\left(x_{m_{k}}, x_{n_{k}}\right) \geq \varepsilon$,

(ii) $\forall k \geq n_{0}, n_{k} \geq m_{k}+2, \mathrm{~d}\left(x_{m_{k}}, x_{n_{k}-1}\right)<\varepsilon$,

(iii) $\mathrm{d}\left(x_{m_{k}}, x_{n_{k}}\right) \underset{k}{\longrightarrow} \varepsilon$.

Proof Since $\mathbb{R}_{+} \backslash \Delta$ is dense in $\mathbb{R}_{+}$and $\left(x_{n}\right)$ is not $\mathrm{f}$-Cauchy, it follows that there exists $\varepsilon>0$ such that, for every $k=1,2, \ldots$, one can find $m, n \in \mathbb{N}, k \leq m<n$, such that $\mathrm{d}\left(x_{m}, x_{n}\right) \geq \varepsilon$.

For each $k \in \mathbb{N}$, we set

$$
\begin{aligned}
& m_{k}=\min \left\{m \in \mathbb{N} ; \exists n \in \mathbb{N}, k \leq m<n, \mathrm{~d}\left(x_{m}, x_{n}\right) \geq \varepsilon\right\}, \\
& n_{k}=\min \left\{n \in \mathbb{N} ; n>m_{k} \text { and } \mathrm{d}\left(x_{m_{k}}, x_{n}\right) \geq \varepsilon\right\} .
\end{aligned}
$$

Hence (i) is satisfied.

As $\mathrm{d}\left(x_{n}, x_{n+1}\right) \rightarrow 0$, there is $n_{0} \in \mathbb{N}$ such that $\mathrm{d}\left(x_{n_{k}}, x_{n_{k}+1}\right)<\varepsilon$ for all $k \geq n_{0}$. So, by the choice of $m_{k}$ and $n_{k}$, for $k \geq n_{0}$ and $n_{k} \geq m_{k}+2$, one has $\mathrm{d}\left(x_{m_{k}}, x_{n_{k}-1}\right)<\varepsilon$, hence (ii).

Next,

$$
\varepsilon \leq \mathrm{d}\left(x_{m_{k}}, x_{n_{k}}\right) \leq \mathrm{d}\left(x_{m_{k}}, x_{n_{k}-1}\right)+\mathrm{d}\left(x_{n_{k}-1}, x_{n_{k}}\right)<\varepsilon+\mathrm{d}\left(x_{n_{k}-1}, x_{n_{k}}\right)
$$

for all $k \geq n_{0}$. Passing to the limit, we get $\lim _{k} \mathrm{~d}\left(x_{m_{k}}, x_{n_{k}}\right)=\varepsilon$.

Definition 3.2 Let $(X, \mathrm{~d})$ be a quasi-metric space and $F: \mathbb{R}_{+} \rightarrow \mathbb{R}$ be a function. A mapping $T: X \rightarrow X$ is said to be a forward F-contraction (resp. backward F-contraction) if there exists $\tau>0$ such that

$$
T x \neq T y \quad \Rightarrow \quad \tau+F(\mathrm{~d}(T x, T y)) \leq F(\mathrm{~d}(x, y))
$$

(resp.

$$
T x \neq T y \quad \Rightarrow \quad \tau+F(\mathrm{~d}(T x, T y)) \leq F(\mathrm{~d}(y, x))) .
$$

We denote by $\mathcal{F}$ the class of all increasing mappings $F: \mathbb{R}_{+} \rightarrow \mathbb{R}$ and, for some $\mu \in \overline{\mathbb{R}}_{+}$, by $\Psi_{\mu}$ the family of all increasing and continuous functions $\psi:(-\infty, \mu) \rightarrow \mathbb{R}$ such that $\psi(t)<t$ for every $t \in(-\infty, \mu)$ (various examples of such functions $\psi$ can be found in [18]).

Notice that every mapping $\psi \in \Psi_{\mu}$ takes values in $(-\infty, \mu)$. 
Lemma 3.2 If $\psi \in \Psi_{\mu}$, then $\psi^{n}(t) \rightarrow-\infty$ for all $t \in(-\infty, \mu)$.

Proof Choose $t \in \mathbb{R}$. From $\psi(t)<t$, one obtains $\psi^{2}(t)<\psi(t)$, and hence the sequence $\left(\psi^{n}(t)\right)$ is decreasing. Let $l \in \mathbb{R} \cup\{-\infty\}$ be its limit. If $l \in \mathbb{R}$, by the continuity of $\psi$ at $l$, it follows $l=\lim _{n} \psi^{n}(t)=\psi(l)<l$, which is a contradiction. Therefore $l=-\infty$.

For a given function $F \in \mathcal{F}$ and $\mu \geq \sup F$, we will write $\Psi$ instead of $\Psi_{\mu}$. Inspired by [18], we will generalize the concept $F$-contraction.

Definition 3.3 Let us consider a quasi-metric space $(X, \mathrm{~d})$ and two functions $F \in \mathcal{F}$ and $\psi \in \Psi$. A mapping $T: X \rightarrow X$ is said to be a forward $\psi F$-contraction (resp. backward $\psi F$-contraction) if

$$
T x \neq T y \quad \Rightarrow \quad F(\mathrm{~d}(T x, T y)) \leq \psi(F(\mathrm{~d}(x, y)))
$$

(resp.

$$
T x \neq T y \quad \Rightarrow \quad F(\mathrm{~d}(T x, T y)) \leq \psi(F(\mathrm{~d}(y, x)))) .
$$

Remark 3.1 If $T$ is a backward $\psi F$-contraction, then $T^{2}$ is a forward $\psi^{2} F$-contraction.

Proof The assertion comes obviously from the following inequalities:

$$
F\left(\mathrm{~d}\left(T^{2} x, T^{2} y\right)\right) \leq \psi(F(\mathrm{~d}(T y, T x))) \leq \psi^{2}(F(\mathrm{~d}(x, y)))
$$

for all $x, y \in X$ such that $T^{2} x \neq T^{2} y$.

It is obvious that every forward $F$-contraction (resp. backward $F$-contraction) is a forward $\psi F$-contraction (resp. backward $\psi F$-contraction) by taking $\psi(t)=t-\tau$.

Remark 3.2 If $T: X \rightarrow X$ is a forward (resp. backward) $\psi F$-contraction, where $F \in \mathcal{F}$, $\psi \in \Psi$, then

$$
\mathrm{d}(T x, T y) \leq \mathrm{d}(x, y) \quad(\text { resp. } \mathrm{d}(T x, T y) \leq \mathrm{d}(y, x)), \quad \forall x, y \in X
$$

hence $T$ is $\mathrm{ff}$ (resp. bf)-continuous.

Proof Choose $x, y \in X$. If $T x=T y$, inequalities (3.3) are obvious. If $T x \neq T y$, by (3.1) and the property of $\psi$, one has

$$
F(\mathrm{~d}(T x, T y)) \leq \psi(F(\mathrm{~d}(x, y)))<F(\mathrm{~d}(x, y)) .
$$

So, $F$ being increasing, the first inequality of (3.3) follows. For the second one, we proceed analogously.

Definition 3.4 A self-mapping $T$ on a quasi-metric space $(X, \mathrm{~d})$ is said to be a forward Picard operator, abbreviated f-P.O. if it has a unique fixed point $\xi \in X$ and $T^{n} x \stackrel{f}{\longrightarrow} \xi$ for every $x \in X$. In a similar way one can define backward Picard operator. 
Theorem 3.1 Let us consider $F \in \mathcal{F}$ and $\psi \in \Psi$. If $T: X \rightarrow X$ is a backward $\psi F$ contraction and the space is $f$-T-orbitally complete, then $T$ is an $f$-P.O.

Proof Let $x_{0} \in X$ and $x_{n}=T^{n} x_{0}, n=1,2, \ldots$. If there exists $n \in \mathbb{N}$ such that $T x_{n}=x_{n}$, then $\xi=x_{n}$ is a fixed point of $T$. Assume that $x_{n+1} \neq x_{n}$. Hence, by (M2), $\mathrm{d}\left(x_{n+1}, x_{n}\right)>0$ and $\mathrm{d}\left(x_{n}, x_{n+1}\right)>0$ for all $n \geq 1$. Then, from (3.2), one has

$$
\begin{aligned}
F\left(\mathrm{~d}\left(x_{n}, x_{n+1}\right)\right) & =F\left(\mathrm{~d}\left(T x_{n-1}, T x_{n}\right)\right) \leq \psi\left(F\left(\mathrm{~d}\left(x_{n}, x_{n-1}\right)\right)\right) \\
& \leq \psi^{2}\left(F\left(\mathrm{~d}\left(x_{n-2}, x_{n-1}\right)\right)\right) \leq \cdots \leq \psi^{n}(F(\gamma)),
\end{aligned}
$$

where $\gamma=\max \left\{\mathrm{d}\left(x_{1}, x_{0}\right), \mathrm{d}\left(x_{0}, x_{1}\right)\right\}$. Consequently, according to Lemma 3.2, $\lim _{n} F\left(\mathrm{~d}\left(x_{n}\right.\right.$, $\left.\left.x_{n+1}\right)\right)=-\infty$ and so, by Lemma 3.1,

$$
\mathrm{d}\left(x_{n}, x_{n+1}\right) \rightarrow 0 .
$$

Analogously one can prove that

$$
\mathrm{d}\left(x_{n+1}, x_{n}\right) \rightarrow 0 .
$$

Arguing by contradiction, we assume that $\left(x_{n}\right)$ is not $\mathrm{f}$-Cauchy. Let us denote by $\Delta$ the set of discontinuities of $F$. Taking into account the monotonicity of $F$, the set $\Delta$ is at most countable, so, as $\mathbb{R}_{+} \backslash \Delta$ is dense in $\mathbb{R}_{+}$, we can apply Proposition 3.1. Thus, we obtain $\varepsilon \in \mathbb{R}_{+} \backslash \Delta$ and sequences of positive integers $\left(m_{k}\right),\left(n_{k}\right)$ such that

$$
\lim _{k} \mathrm{~d}\left(x_{m_{k}}, x_{n_{k}}\right)=\varepsilon
$$

We have

$$
\mathrm{d}\left(x_{m_{k}}, x_{n_{k}}\right)-\mathrm{d}\left(x_{m_{k}}, x_{m_{k}+1}\right)-\mathrm{d}\left(x_{n_{k}+1}, x_{n_{k}}\right) \leq \mathrm{d}\left(x_{m_{k}+1}, x_{n_{k}+1}\right)
$$

and

$$
\mathrm{d}\left(x_{m_{k}-1}, x_{n_{k}-1}\right) \leq \mathrm{d}\left(x_{m_{k}-1}, x_{m_{k}}\right)+\mathrm{d}\left(x_{m_{k}}, x_{n_{k}}\right)+\mathrm{d}\left(x_{n_{k}}, x_{n_{k}-1}\right) .
$$

Since by (3.4), (3.5), and (3.6) one has

$$
\mathrm{d}\left(x_{m_{k}}, x_{n_{k}}\right)-\mathrm{d}\left(x_{m_{k}}, x_{m_{k}+1}\right)-\mathrm{d}\left(x_{n_{k}+1}, x_{n_{k}}\right) \rightarrow \varepsilon>0,
$$

one can find $K \in \mathbb{N}$ such that

$$
\mathrm{d}\left(x_{m_{k}}, x_{n_{k}}\right)-\mathrm{d}\left(x_{m_{k}}, x_{m_{k}+1}\right)-\mathrm{d}\left(x_{n_{k}+1}, x_{n_{k}}\right)>0
$$

for all $k \geq K$.

Using now (3.9), (3.7), (3.2), (3.8) and since $F$ is increasing, it follows

$$
\begin{aligned}
& F\left(\mathrm{~d}\left(x_{m_{k}}, x_{n_{k}}\right)-\mathrm{d}\left(x_{m_{k}}, x_{m_{k}+1}\right)-\mathrm{d}\left(x_{n_{k}+1}, x_{n_{k}}\right)\right) \\
& \quad \leq F\left(\mathrm{~d}\left(x_{m_{k}+1}, x_{n_{k}+1}\right)\right)
\end{aligned}
$$




$$
\begin{aligned}
& \leq \psi\left(F\left(\mathrm{~d}\left(x_{n_{k}}, x_{m_{k}}\right)\right)\right) \leq \psi^{2}\left(F\left(\mathrm{~d}\left(x_{m_{k}-1}, x_{n_{k}-1}\right)\right)\right) \\
& \leq \psi^{2}\left(F\left(\mathrm{~d}\left(x_{m_{k}-1}, x_{m_{k}}\right)+\mathrm{d}\left(x_{m_{k}}, x_{n_{k}}\right)+\mathrm{d}\left(x_{n_{k}}, x_{n_{k}-1}\right)\right)\right), \quad \forall k \geq K .
\end{aligned}
$$

By letting $k \rightarrow \infty$ in the above relations and using the continuity of $F$ at $\varepsilon$, the continuity of $\psi$, and relations (3.4), (3.5), and (3.6), one obtains

$$
F(\varepsilon) \leq \psi^{2}(F(\varepsilon))<F(\varepsilon)
$$

which is a contradiction.

Consequently, $\left(x_{n}\right)$ is f-Cauchy so, by hypothesis, there exists $\xi \in X$ such that $x_{n} \stackrel{f}{\longrightarrow} \xi$, that is, $\mathrm{d}\left(\xi, x_{n}\right) \rightarrow 0$. We will show that $\xi$ is a fixed point for $T$.

From (3.2) and since $F$ and $\psi$ are increasing, clearly

$$
\mathrm{d}(T x, T y) \leq \mathrm{d}(y, x), \quad \forall x, y \in X
$$

Hence we get

$$
\mathrm{d}(\xi, T \xi) \leq \mathrm{d}\left(\xi, x_{n}\right)+\mathrm{d}\left(x_{n}, T \xi\right)=\mathrm{d}\left(\xi, x_{n}\right)+\mathrm{d}\left(T x_{n-1}, T \xi\right) \leq \mathrm{d}\left(\xi, x_{n}\right)+\mathrm{d}\left(\xi, x_{n-1}\right) .
$$

By letting $n \rightarrow \infty$ in the previous relations, it follows $\mathrm{d}(\xi, T \xi)=0$ so, from (M2), $T \xi=\xi$.

In order to prove the uniqueness, assume by contradiction that there is $\eta \in X, \eta \neq \xi$ such that $T \eta=\eta$. Then $T \eta \neq T \xi$ and

$$
\begin{aligned}
F(\mathrm{~d}(\eta, \xi)) & =F(\mathrm{~d}(T \eta, T \xi)) \leq \psi(F(\mathrm{~d}(\xi, \eta))) \\
& =\psi(F(\mathrm{~d}(T \xi, T \eta))) \leq \psi^{2}(F(\mathrm{~d}(\eta, \xi)))<F(\mathrm{~d}(\eta, \xi)),
\end{aligned}
$$

which is a contradiction.

The proof is complete.

Corollary 3.1 Assume that the quasi-metric space $(X, \mathrm{~d})$ is $f$-complete, $F \in \mathcal{F}$ and $\psi \in \Psi$. If $T: X \rightarrow X$ is a backward $\psi F$-contraction, then $T$ is an $f$-P.O.

Remark 3.3 The above corollary generalizes and improves [13, Th. 2.2], where $F(t)=\ln t$ and $\psi(t)=t-\tau, \tau>0$.

Example 3.1 Let us consider $X=[1, \infty)$ and the mapping $\mathrm{d}: X \times X \rightarrow \mathbb{R}_{+} \cup\{0\}$ given as

$$
\mathrm{d}(x, y)= \begin{cases}\ln y-\ln x, & \text { if } y \geq x, \\ \frac{1}{2}(\ln x-\ln y), & \text { if } y<x .\end{cases}
$$

We also define $F: \mathbb{R}_{+} \rightarrow \mathbb{R}, F(t)=\frac{1}{1-e^{t}}$, and $\psi:(-\infty, 0) \rightarrow \mathbb{R}, \psi(t)=t-\frac{\lambda}{\pi} \operatorname{arccot} t+\lambda-$ 1 , where $0 \leq \lambda<1$. Then $\mathrm{d}$ is a quasi-metric on $X, F \in \mathcal{F}, \psi \in \Psi$ and the mapping $T$ : $X \rightarrow X, T x=\frac{x+1}{x}$ is a backward $\psi F$-contraction on $(X, \mathrm{~d})$, whereas it is not forward $\psi F$ contraction. Moreover, $T$ is an f-P.O. 
Proof It is easy to check that $\mathrm{d}$ is a quasi-metric on $X$. One can also simply see that $F$ and $\psi$ are increasing and $\sup F=0$.

We first show (3.2). Since $\psi(t) \geq t-1$, for all $t<0$, it is enough to prove that

$$
T x \neq T y \quad \Rightarrow \quad F(\mathrm{~d}(T x, T y)) \leq F(\mathrm{~d}(y, x))-1
$$

For this purpose, choose $x, y \in X, x \neq y$.

If $x<y$, one has $T x>T y$ so

$$
\mathrm{d}(y, x)=\ln \frac{\sqrt{y}}{\sqrt{x}}, \quad \mathrm{~d}(T x, T y)=\ln \frac{\sqrt{T x}}{\sqrt{T y}}=\ln \frac{\sqrt{y(x+1)}}{\sqrt{x(y+1)}} .
$$

Hence

$$
\begin{aligned}
F(\mathrm{~d}(y, x))-F(\mathrm{~d}(T x, T y)) & =\frac{\sqrt{x}}{\sqrt{x}-\sqrt{y}}-\frac{\sqrt{x(y+1)}}{\sqrt{x(y+1)}-\sqrt{y(x+1)}} \\
& =\frac{\sqrt{x y}(\sqrt{y+1}-\sqrt{x+1})}{(\sqrt{y}-\sqrt{x})(\sqrt{y(x+1)}-\sqrt{x(y+1)})} .
\end{aligned}
$$

Some elementary computations lead to

$$
\frac{\sqrt{x y}}{\sqrt{y}-\sqrt{x}} \geq 1 \text { and } \frac{\sqrt{y+1}-\sqrt{x+1}}{\sqrt{y(x+1)}-\sqrt{x(y+1)}} \geq 1 .
$$

Thus we obtain (3.10).

Assume that $x>y$. Then $T x<T y$ and

$$
\mathrm{d}(y, x)=\ln \frac{x}{y}, \quad \mathrm{~d}(T x, T y)=\ln \frac{y+1}{x+1} \cdot \frac{x}{y} .
$$

Consequently,

$$
F(\mathrm{~d}(y, x))-F(\mathrm{~d}(T x, T y))=\frac{y}{y-x}-\frac{y(x+1)}{y-x}=\frac{x y}{x-y} \geq 1
$$

so (3.10) is verified.

We now prove that $T$ is not forward $\psi F$-contraction. Assume by contradiction that (3.1) holds. Then

$$
T x \neq T y \quad \Rightarrow \quad F(\mathrm{~d}(T x, T y)) \leq F(\mathrm{~d}(x, y))-\frac{\lambda}{\pi} \operatorname{arccot} \mathrm{d}(x, y)+\lambda-1,
$$

that is,

$$
F(\mathrm{~d}(x, y))-F(\mathrm{~d}(T x, T y)) \geq \frac{\lambda}{\pi} \operatorname{arccot} \mathrm{d}(x, y)+1-\lambda>\frac{\lambda}{\pi} \cdot \frac{\pi}{2}+1-\lambda=1-\frac{\lambda}{2} .
$$

Set $y=1$. Then, for each $x>y$,

$$
F(\mathrm{~d}(x, 1))-F(\mathrm{~d}(T x, T 1))=\frac{1}{1-\sqrt{x}}-\frac{x+1}{1-x}=\frac{\sqrt{x}}{\sqrt{x}+1} .
$$


Since $\lim _{x \searrow 1} \frac{\sqrt{x}}{\sqrt{x}+1}=\frac{1}{2}$ and, by hypothesis, $1-\frac{\lambda}{2}>\frac{1}{2}$, one can find $x>1$ such that $\frac{\sqrt{x}}{\sqrt{x}+1}<$ $1-\frac{\lambda}{2}$. This contradicts (3.11).

Therefore $T$ is not forward $\psi F$-contraction.

In order to prove the forward completeness of $X$, let $\left(x_{n}\right)$ be a forward Cauchy sequence and fix $\varepsilon>0$. Then there exists $N \in \mathbb{N}$ such that $\mathrm{d}\left(x_{n}, x_{m}\right)<\frac{\varepsilon}{2}$ for every $m \geq n \geq N$. It is easy to see that this sequence is forward bounded. Since the topology induced by $\mathrm{d}$ is the Euclidean one, this implies forward convergence of a subsequence $\left(x_{n_{k}}\right)$ to some $x \in X$. Choose $k \in \mathbb{N}$ so that $n_{k} \geq N$ and $\mathrm{d}\left(x, x_{n_{k}}\right)<\frac{\varepsilon}{2}$. Then, for $n \geq n_{k} \geq N$, one has

$$
\mathrm{d}\left(x, x_{n}\right) \leq \mathrm{d}\left(x, x_{n_{k}}\right)+\mathrm{d}\left(x_{n_{k}}, x_{n}\right)<\varepsilon
$$

so $x_{n} \stackrel{f}{\longrightarrow} x$. Consequently $(X, \mathrm{~d})$ is forward complete.

The final conclusion now follows from Corollary 3.1 .

Notice that some examples of forward $\psi F$-contractions can be found in Example 4.1.

Corollary 3.2 If $(X, \mathrm{~d})$ is $f$-complete and $f$-convergence implies b-convergence, then every forward $\psi F$-contraction $T: X \rightarrow X$ is an f-P.O., where $F \in \mathcal{F}, \psi \in \Psi$.

Proof Obvious.

Remark 3.4 The above corollary generalizes and improves [13, Th. 2.1], where $F(t)=\ln t$ and $\psi(t)=t-\tau, \tau>0$.

Theorem 3.2 Let us consider $F \in \mathcal{F}, \psi \in \Psi$ and a backward $\psi F$-contraction $T: X \rightarrow X$. If there exists $x_{0} \in X$ such that the sequence $\left(T^{n} x_{0}\right)$ is f-convergent, then $T$ is an f-P.O.

Proof Let $\xi \in X$ be such that $\lim _{n} \mathrm{~d}\left(\xi, T^{n} x_{0}\right)=0$. Then

$$
\mathrm{d}(\xi, T \xi) \leq \mathrm{d}\left(\xi, T^{n} x_{0}\right)+\mathrm{d}\left(T^{n} x_{0}, T \xi\right) \leq \mathrm{d}\left(\xi, T^{n} x_{0}\right)+\mathrm{d}\left(\xi, T^{n-1} x_{0}\right) \rightarrow 0,
$$

and so $T \xi=\xi$.

Choose $y_{0} \in X$. If there is $n \in \mathbb{N}$ such that $T^{n} y_{0}=T^{n} x_{0}$, then $T^{n} y_{0} \stackrel{f}{\longrightarrow} \xi$. Otherwise one has

$$
F\left(\mathrm{~d}\left(T^{n} x_{0}, T^{n} y_{0}\right)\right) \leq \psi\left(F\left(\mathrm{~d}\left(T^{n-1} y_{0}, T^{n-1} x_{0}\right)\right)\right) \leq \cdots \leq \psi^{n}(F(\gamma)) \underset{n}{\longrightarrow}-\infty,
$$

where $\gamma=\max \left\{\mathrm{d}\left(x_{0}, y_{0}\right), \mathrm{d}\left(y_{0}, x_{0}\right)\right\}$. So, by Lemma 3.1, $\mathrm{d}\left(T^{n} x_{0}, T^{n} y_{0}\right) \rightarrow 0$. Thus

$$
\mathrm{d}\left(\xi, T^{n} y_{0}\right) \leq \mathrm{d}\left(\xi, T^{n} x_{0}\right)+\mathrm{d}\left(T^{n} x_{0}, T^{n} y_{0}\right) \rightarrow 0
$$

Consequently, $T^{n} y_{0} \stackrel{f}{\longrightarrow} \xi$.

For the uniqueness of the fixed point of $T$, we proceed as in Theorem 3.1.

Theorem 3.3 Take any $F \in \mathcal{F}$ and $\psi \in \Psi$. Assume that $T: X \rightarrow X$ is a forward $\psi F$ contraction and the space is forward T-orbitally complete. If one of the following sentences holds:

(a) $T$ is fb-orbitally continuous; 
(b) the space $(X, \mathrm{~d})$ is Hausdorff;

(c) in the space $(X, \mathrm{~d}) f$-convergence implies b-convergence;

then $T$ is an $f$-P.O.

Proof Set $x_{0} \in X$ and $x_{n}=T^{n} x_{0}, n=1,2, \ldots$. For the Cauchyness and uniqueness, we can proceed in the same manner as in the proof of Theorem 3.1.

In case (a), as $x_{n}=T^{n} x_{0} \stackrel{f}{\longrightarrow} \xi$ one has $T x_{n}=T^{n+1} x_{0} \stackrel{b}{\longrightarrow} T \xi$, that is, $\mathrm{d}\left(T x_{n}, T \xi\right) \rightarrow 0$. Hence,

$$
\mathrm{d}(\xi, T \xi) \leq \mathrm{d}\left(\xi, x_{n+1}\right)+\mathrm{d}\left(T x_{n}, T \xi\right) \rightarrow 0
$$

so $T \xi=\xi$.

In case (b), using (3.1), we have $\mathrm{d}\left(T \xi, x_{n}\right)=\mathrm{d}\left(T \xi, T x_{n-1}\right) \leq \mathrm{d}\left(\xi, x_{n-1}\right)$ for all $n \geq 1$, therefore $x_{n} \stackrel{f}{\longrightarrow} T \xi$. The space being Hausdorff, we get $T \xi=\xi$.

In case (c) the conclusion follows from (b) and Proposition 2.1.

\subsection{Space of fractals}

The concept "space of fractals" was introduced by Barnsley in his famous book [28] to denote the class of all non-empty compact subsets of a metric space endowed with the Hausdorff-Pompeiu metric. For completeness we will describe some basic concepts and results in the settings of quasi-metric spaces even though some of proofs are similar to those in metric spaces.

Let us denote by $\mathcal{P}_{\mathrm{f}}(X)$, respectively $\mathcal{P}_{\mathrm{b}}(X), \mathcal{P}_{\mathrm{fb}}(X)$, the family of all non-empty and $\mathrm{f}$ bounded, respectively b-bounded, fb-bounded, subsets of the quasi-metric space $(X, \mathrm{~d})$. We also denote, for every $A, B \in \mathcal{P}_{\mathrm{b}}(X)$,

$$
\mathrm{D}(A, B):=\sup _{x \in A} \inf _{y \in B} \mathrm{~d}(x, y) \quad \text { and } \quad h(A, B):=\max \{\mathrm{D}(A, B), \mathrm{D}(B, A)\} .
$$

Remark 3.5 The condition $A, B$ b-bounded in definition of $\mathrm{D}$ is necessary to have $\mathrm{D}(A, B)<$ $\infty$. This inequality is not true if we suppose that $A, B$ are $\mathrm{f}$-bounded.

Proof Indeed, assume that $A \neq \emptyset$ is b-bounded and $\emptyset \neq B \subset X$. Arguing by contradiction, assume that

$$
\mathrm{D}(A, B)=\sup _{x \in A} \inf _{y \in B} \mathrm{~d}(x, y)=\infty .
$$

Hence, for each $n=1,2, \ldots$, one can find $x_{n} \in A$ such that $\inf _{y \in B} \mathrm{~d}\left(x_{n}, y\right) \geq n$. So $\mathrm{d}\left(x_{n}, y\right) \geq n$ for all $n \in \mathbb{N}$ and all $y \in B$. Therefore, for some $y \in B$, we have $\sup _{x \in A} \mathrm{~d}(x, y)=\infty$ contradicting the b-boundedness of $A$.

In order to prove the second part of the statement, we give a contra-example. In the quasi-metric space $(\mathbb{R}, \mathrm{d})$ from Example 2.4, the sets $A=(-\infty, a]$ and $B=\{b\}, a, b \in \mathbb{R}$, are $\mathrm{f}$-closed and f-bounded. However $\mathrm{D}(A, B)=\infty$.

Indeed, it is clear that $A$ and $B$ are $\mathrm{f}$-closed and $B$ is f-bounded. Since $\sup _{y \in A} \mathrm{~d}(a, y)=1$, it follows that $A$ is $\mathrm{f}$-bounded. Next,

$$
\mathrm{D}(A, B)=\sup _{x \in A} \inf _{y \in B} \mathrm{~d}(x, y)=\sup _{x \in A} \mathrm{~d}(x, b)=\sup _{x \in A}(b-x)=\infty .
$$


In the following some basic properties of the functions $\mathrm{D}$ and $h$ are highlighted.

\section{Proposition 3.2 The following assertions hold:}

(a) $A \subset B \Rightarrow D(A, B)=0$; in particular $\mathrm{D}(A, A)=0$ for all $A \in \mathcal{P}_{\mathrm{b}}(X)$;

(b) $\mathrm{D}(A, B)=0 \Rightarrow A \subset \bar{B}$, where the bar means the closure in the topological space $\left(X, \mathcal{T}_{f}\right)$

(c) $\mathrm{D}(A, C) \leq \mathrm{D}(A, B)+\mathrm{D}(B, C)$ for all $A, B, C \in \mathcal{P}_{\mathrm{b}}(X)$;

(d) $\mathrm{D}(A, B)=\mathrm{D}(\bar{A}, \bar{B})$ for all $A, B \in \mathcal{P}_{\mathrm{b}}(X)$;

(e) $\mathrm{D}\left(\bigcup_{i \in \mathfrak{\Im}} A_{i}, \bigcup_{i \in \mathfrak{\Im}} B_{i}\right) \leq \sup _{i \in \mathfrak{\Im}} \mathrm{D}\left(A_{i}, B_{i}\right)$ for every family of sets $\left(A_{i}\right),\left(B_{i}\right)$ such that $\bigcup_{i \in \mathfrak{\Im}} A_{i}$ and $\bigcup_{i \in \Im} B_{i}$ are b-bounded.

Proof (a) If $A \subset B$ and $x \in A$, then $\inf _{y \in B} \mathrm{~d}(x, y)=0$ because $x \in B$ and $\mathrm{d}(x, x)=0$. Hence $\sup _{x \in A} \inf _{y \in A} \mathrm{~d}(x, y)=0$.

(b) Assume that $A, B \in \mathcal{P}_{\mathrm{b}}(X)$, $\sup _{x \in A} \inf _{y \in B} \mathrm{~d}(x, y)=0$. Choose $x \in A$. Then $\inf _{y \in B} \mathrm{~d}(x, y)=$ 0 , so, for each $n \in \mathbb{N}$, there is $y_{n} \in B$ such that $\mathrm{d}\left(x, y_{n}\right)<\frac{1}{n}$. Thus $y_{n} \stackrel{f}{\rightarrow} x$, that is, $x \in \bar{B}$.

(c) For $x \in A, z \in C$, one has

$$
\mathrm{d}(x, z) \leq \mathrm{d}(x, y)+\mathrm{d}(y, z), \quad \forall y \in B .
$$

So

$$
\begin{aligned}
& \inf _{z \in C} \mathrm{~d}(x, z) \leq \inf _{z \in C}(\mathrm{~d}(x, y)+\mathrm{d}(y, z))=\mathrm{d}(x, y)+\inf _{z \in C} \mathrm{~d}(y, z), \quad \forall y \in B \\
& \Rightarrow \quad \inf _{z \in C} \mathrm{~d}(x, z) \leq \inf _{y \in B}\left(\mathrm{~d}(x, y)+\inf _{z \in C} \mathrm{~d}(y, z)\right) \\
& \leq \inf _{y \in B} \mathrm{~d}(x, y)+\sup _{y \in B} \inf _{z \in C} \mathrm{~d}(y, z)=\inf _{y \in B} \mathrm{~d}(x, y)+\mathrm{D}(B, C) .
\end{aligned}
$$

Since $x$ is arbitrarily chosen, we get the inequality from the statement.

(d) Let us consider $A, B \in \mathcal{P}_{\mathrm{b}}(X)$. From (a) it follows $\mathrm{D}(A, \bar{A})=0$ and $\mathrm{D}(B, \bar{B})=0$ because $A \subset \bar{A}$ and $B \subset \bar{B}$. We will show that $\mathrm{D}(\bar{A}, A)=0$. Indeed, on the contrary, one can find $\lambda>0$ such that $\mathrm{D}(\bar{A}, A)=\sup _{x \in \bar{A}} \inf _{y \in A} \mathrm{~d}(x, y)>\lambda$. Then there exists $x \in \bar{A}$ such that $\inf _{y \in A} \mathrm{~d}(x, y)>\lambda$, so

$$
\mathrm{d}(x, y)>\lambda, \quad \forall y \in A .
$$

Next, there is a sequence $\left(x_{n}\right) \subset A$ such that $x_{n} \stackrel{f}{\longrightarrow} x$, that is, $\mathrm{d}\left(x, x_{n}\right) \rightarrow 0$ which contradicts (3.13). Thus $\mathrm{D}(\bar{A}, A)=0$. Analogously we can prove that $\mathrm{D}(\bar{B}, B)=0$.

Using now (c) and the previous equalities, one has

$$
\begin{aligned}
& \mathrm{D}(A, B) \leq \mathrm{D}(A, \bar{A})+\mathrm{D}(\bar{A}, \bar{B})+\mathrm{D}(\bar{B}, B)=\mathrm{D}(\bar{A}, \bar{B}), \\
& \mathrm{D}(\bar{A}, \bar{B}) \leq \mathrm{D}(\bar{A}, A)+\mathrm{D}(A, B)+\mathrm{D}(B, \bar{B})=\mathrm{D}(A, B),
\end{aligned}
$$

consequently $\mathrm{D}(A, B)=\mathrm{D}(\bar{A}, \bar{B})$.

(e) Choose $x \in \bigcup_{i \in \mathfrak{s}} A_{i}$. There is $i_{x} \in \Im$ such that $x \in A_{i_{x}}$. We have

$$
\inf _{y \in \bigcup_{i \in \mathfrak{\Im}} B_{i}} \mathrm{~d}(x, y) \leq \inf _{y \in B_{i_{x}}} \mathrm{~d}(x, y) \leq \mathrm{D}\left(A_{i_{x}}, B_{i_{x}}\right) \leq \sup _{i \in \mathfrak{\Im}} \mathrm{D}\left(A_{i}, B_{i}\right) .
$$

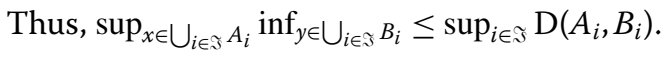


According to the previous proposition, we can easily obtain the following properties for $h$.

Corollary 3.3 The following assertions hold:

(a) $h(A, B)=0 \Leftrightarrow \bar{A}=\bar{B}$;

(b) $h(A, C) \leq h(A, B)+h(B, C)$ for all $A, B, C \in \mathcal{P}(X)$;

(c) $h(A, B)=h(\bar{A}, \bar{B})$ for all $A, B \in \mathcal{P}_{\mathrm{b}}(X)$;

(d) $h\left(\overline{\bigcup_{i \in \mathfrak{I}} A_{i}}, \overline{\bigcup_{i \in \Im} B_{i}}\right)=h\left(\bigcup_{i \in \Im} A_{i}, \bigcup_{i \in \mathfrak{\Im}} B_{i}\right) \leq \sup _{i \in \mathfrak{\Im}} h\left(A_{i}, B_{i}\right)$ for every family of sets $\left(A_{i}\right),\left(B_{i}\right)$ such that $\bigcup_{i \in \Im} A_{i}$ and $\bigcup_{i \in \mathfrak{s}} B_{i}$ are b-bounded.

In view of the aforesaid, the function $h$ is a pseudo-metric on $\mathcal{P}_{\mathrm{b}}(X)$. Furthermore, if we denote by $\mathcal{C}_{\mathrm{f}}(X)$ the class of all non-empty, b-bounded, and f-closed subsets of $X$, then the mapping $h$ is a metric on $\mathcal{C}_{\mathrm{f}}(X)$ called $f$-Hausdorff-Pompeiu metric.

For $A, B \in \mathcal{P}_{\mathrm{f}}(X)$, let us denote $\overline{\mathrm{D}}(A, B)=\sup _{x \in A} \inf _{y \in B} \mathrm{~d}(y, x)$ and

$$
\begin{aligned}
& h_{1}(A, B)=\max \{\mathrm{D}(A, B), \overline{\mathrm{D}}(B, A)\}, \\
& h_{2}(A, B)=\max \{\overline{\mathrm{D}}(A, B), \mathrm{D}(B, A)\}, \\
& h_{3}(A, B)=\max \{\overline{\mathrm{D}}(A, B), \overline{\mathrm{D}}(B, A)\} .
\end{aligned}
$$

In a similar manner as in Proposition 3.2 one can prove analogous properties for $\overline{\mathrm{D}}$. Denoting by $\mathcal{C}_{\mathrm{b}}(X)$ the class of all non-empty, f-bounded, and b-closed subsets of $X$, it follows that $h_{1}$ and $h_{2}$ are quasi-metrics on $\mathcal{C}_{\mathrm{f}}(X) \cap \mathcal{C}_{\mathrm{b}}(X)$ called fb-Hausdorff-Pompeiu and bf-Hausdorff-Pompeiu quasi-metric, respectively. Likewise $h_{3}$ is a metric on $\mathcal{C}_{\mathrm{b}}(X)$, named b-Hausdorff-Pompeiu metric (for a some approach in this setting, see also [9]).

For simplicity, we will work in the sequel only with $h$.

We denote by $\mathcal{K}_{\mathrm{f}}(X)$ the family of all non-empty f-compact subsets of $X$.

If in the quasi-metric space $(X, \mathrm{~d}) \mathrm{f}$-convergence implies b-convergence, we have seen (Proposition 2.1) that $\left(X, \mathcal{T}_{\mathrm{f}}\right)$ is Hausdorff. Hence it is well known that in this space every compact set is f-closed. Thus, according to Proposition 2.3, we conclude that $\mathcal{K}_{\mathrm{f}}(X) \subset$ $\mathcal{C}_{\mathrm{f}}(X)$.

Definition 3.5 In the above settings, the metric space $\left(\mathcal{K}_{\mathrm{f}}(X), h\right)$ is called fractals space.

Lemma 3.3 Assume that the quasi-metric space $(X, \mathrm{~d})$ is $f$-complete and $\left(F_{n}\right)$ is a decreasing sequence of non-empty $f$-closed subsets of $X$. Then $\bigcap_{n=1}^{\infty} F_{n} \neq \emptyset$.

Proof For each $n=1,2, \ldots$, set $\lambda_{n}:=\operatorname{diam}\left(F_{n}\right)=\sup _{x, y \in F} \mathrm{~d}(x, y) \in \mathbb{R}_{+} \cup\{\infty\}$. Since $\left(F_{n}\right)$ is decreasing, it follows that $\left(\lambda_{n}\right)$ decreases to a limit $\lambda \in \mathbb{R}_{+} \cup\{\infty\}$.

If $\lambda>0$, then clearly $\bigcap_{n=1}^{\infty} F_{n} \neq \emptyset$.

Assume that $\lambda=0$ and, for each $n \in \mathbb{N}$, choose $x_{n} \in F_{n}$. Then, for $\varepsilon>0$, one can find $N \in \mathbb{N}$ such that $\operatorname{diam}\left(F_{N}\right)<\varepsilon$. Since $\left(F_{n}\right)$ is decreasing, $x_{n} \in F_{N}$ for all $n \geq N$. Hence, for every $m>n \geq N$, we have $\mathrm{d}\left(x_{n}, x_{m}\right) \leq \operatorname{diam}\left(F_{N}\right)<\varepsilon$. Consequently the sequence $\left(x_{n}\right)$ is $\mathrm{f}$-Cauchy so, the space being $\mathrm{f}$-complete, it is convergent. Let $x \in X$ be its limit. Now, since, for every $n \in \mathbb{N},\left(x_{k}\right)_{k \geq n} \subset F_{n}$ and $F_{n}$ is f-closed, it follows that $x \in F_{n}$. Accordingly, $x \in \bigcap_{n=1}^{\infty} F_{n}$ completing the proof. 
Notice that a Cantor type theorem for characterization of completeness in quasi-metric spaces can be found in [16, Th. 10]. The above result is appropriate to our purpose.

Lemma 3.4 Let $\left(A_{n}\right)$ be a Cauchy sequence in the metric space $\left(\mathcal{C}_{\mathrm{f}}(X), h\right)$ and $A:=$ $\bigcap_{k=1}^{\infty} \overline{\bigcup_{n \geq k} A_{n}}$. Then $A \in \mathcal{C}_{\mathrm{f}}(X)$.

If, in addition, $f$-convergence is equivalent to b-convergence and $\left(A_{n}\right) \subset \mathcal{K}_{\mathrm{f}}(X)$, then $A \in$ $\mathcal{K}_{\mathrm{f}}(X)$.

Proof According to Lemma 3.3, we have $A \neq \emptyset$. Clearly $A$ is f-closed.

In order to prove the b-boundedness of $A$, fix $\varepsilon>0$. There exists $N \in \mathbb{N}$ such that

$$
h\left(A_{n}, A_{m}\right)<\varepsilon, \quad \forall m, n \geq N .
$$

We claim that $\mathrm{D}\left(A, A_{N}\right) \leq \varepsilon$. Indeed, by (3.14) and Proposition 3.2(d), (e), one has

$$
\mathrm{D}\left(A, A_{N}\right) \leq \mathrm{D}\left(\overline{\bigcup_{n \geq N} A_{n}}, A_{N}\right)=\mathrm{D}\left(\bigcup_{n \geq N} A_{n}, A_{N}\right) \leq \sup _{n \geq N} \mathrm{D}\left(A_{n}, A_{N}\right) \leq \varepsilon .
$$

That is, $\sup _{x \in A} \inf _{y \in A_{N}} \mathrm{~d}(x, y) \leq \varepsilon$ so, for each $x \in A$, there is $y \in A_{N}$ such that $\mathrm{d}(x, y) \leq \varepsilon$.

As $A_{N}$ is b-bounded, for some $z \in X$, one has $\sup _{y \in A_{N}} \mathrm{~d}(y, z)<\infty$. Hence, for every $x \in A$, there exists $y \in A_{N}$ such that

$$
\mathrm{d}(x, z) \leq \mathrm{d}(x, y)+\mathrm{d}(y, z) \leq \varepsilon+\mathrm{d}(y, z) .
$$

Therefore $\sup _{x \in A} \mathrm{~d}(x, z) \leq \varepsilon+\sup _{y \in A_{N}} \mathrm{~d}(y, z)<\infty$, so $A$ is b-bounded.

For the second assertion of the statement, assume that $\mathrm{f}$-convergence is equivalent to b-convergence and $\left(A_{n}\right)$ is a Cauchy sequence in $\left(\mathcal{K}_{\mathrm{f}}(X), h\right)$. Proceeding as above, for some $\varepsilon>0$, there exists $N \in \mathbb{N}$ such that, for each $x \in A$, one can find $y \in A_{N}$ such that $\mathrm{d}(x, y)<\frac{\varepsilon}{2}$. We will prove that $A$ is f-sequentially compact. For this purpose, let $\left(x_{n}\right) \subset A$. Then, as before, for $n=1,2, \ldots$, there exists $y_{n} \in A_{N}$ such that

$$
\mathrm{d}\left(x_{n}, y_{n}\right)<\frac{\varepsilon}{2}
$$

Since, by Lemma 2.1, $A_{N}$ is f-sequentially compact, there exists a subsequence $\left(y_{n_{k}}\right)_{k}$ and $y \in A_{N}$ such that $y_{n_{k}} \stackrel{f}{\longrightarrow} y$ so, from hypothesis, $y_{n_{k}} \stackrel{b}{\longrightarrow} y$. Therefore one can find $K \in \mathbb{N}$ such that

$$
\mathrm{d}\left(y_{n_{k}}, y\right)<\frac{\varepsilon}{2}, \quad \forall k \geq K
$$

We conclude from (3.15) and (3.16) that

$$
\mathrm{d}\left(x_{n_{k}}, y\right) \leq \mathrm{d}\left(x_{n_{k}}, y_{n_{k}}\right)+\mathrm{d}\left(y_{n_{k}}, y\right)<\varepsilon
$$

for every $k \geq K$ for which $n_{k} \geq N$. Thus $x_{n_{k}} \stackrel{b}{\longrightarrow} y$. By hypothesis, one has $x_{n_{k}} \stackrel{f}{\longrightarrow} y$, which proves that $A$ is $\mathrm{f}$-sequentially compact. The conclusion now follows from Corollary 2.1. 
Theorem 3.4 If $(X, \mathrm{~d})$ is $f$-complete and $f$-convergence implies b-convergence, then $\left(\mathcal{C}_{\mathrm{f}}(X)\right.$, $h)$ is complete. If, in addition, b-convergence implies f-convergence, then $\left(\mathcal{K}_{\mathrm{f}}(X), h\right)$ is complete.

Proof Let $\left(A_{n}\right) \subset \mathcal{C}_{\mathrm{f}}(X)$, respectively $\left(A_{n}\right) \subset \mathcal{K}_{\mathrm{f}}(X)$, be a Cauchy sequence in the space $\left(\mathcal{C}_{\mathrm{f}}(X), h\right)$, respectively $\left(\mathcal{K}_{\mathrm{f}}(X), h\right)$, and set $A:=\bigcap_{k=1}^{\infty} \overline{\bigcup_{n \geq k} A_{n}}$.

By Lemma 3.4 $A \in \mathcal{C}_{\mathrm{f}}(X)$, respectively $A \in \mathcal{K}_{\mathrm{f}}(X)$.

We intend to prove that $A_{n} \rightarrow A$ with respect to the f-Hausdorff-Pompeiu metric.

Fix $\varepsilon>0$. There exists $N(\varepsilon) \in \mathbb{N}$ such that

$$
h\left(A_{n}, A_{m}\right)<\frac{\varepsilon}{2}, \quad \forall m, n \geq N(\varepsilon) .
$$

We proceed to show that

$$
h\left(A, A_{m}\right) \leq \varepsilon, \quad \forall m \geq N(\varepsilon) .
$$

The proof of this fact will be divided into two steps.

Step I $\mathrm{D}\left(A, A_{m}\right) \leq \frac{\varepsilon}{3}$ for all $m \geq N(\varepsilon)$.

According to Proposition 3.2(d), (e), and (3.17), we have

$$
\mathrm{D}\left(A, A_{m}\right) \leq \mathrm{D}\left(\overline{\bigcup_{n \geq N(\varepsilon)} A_{n}}, A_{m}\right)=\mathrm{D}\left(\bigcup_{n \geq N(\varepsilon)} A_{n}, A_{m}\right) \leq \sup _{n \geq N(\varepsilon)} \mathrm{D}\left(A_{n}, A_{m}\right) \leq \frac{\varepsilon}{3} .
$$

Step II $\mathrm{D}\left(A_{m}, A\right) \leq \varepsilon$ for all $m \geq N(\varepsilon)$.

Choose $m \geq N(\varepsilon)$ and $y \in A_{m}$. For each $k=0,1,2, \ldots$, let $n_{k}:=N\left(\frac{\varepsilon}{2^{k}}\right)$. We construct the sequence $\left(y_{n_{k}}\right)_{k}$ as follows: $y_{n_{0}}:=y$. Assume that we have $y_{n_{i}} \in A_{n_{i}}, i=1, \ldots, k-1$. Next, taking $\frac{\varepsilon}{2^{k-1}}$ instead of $\varepsilon$, relation (3.17) becomes

$$
h\left(A_{n_{k-1}}, A_{n_{k}}\right)<\frac{\varepsilon}{3 \cdot 2^{k-1}} .
$$

As $y_{n_{k-1}} \in A_{n_{k-1}}$, one can find $y_{n_{k}} \in A_{n_{k}}$ such that

$$
\mathrm{d}\left(y_{n_{k-1}}, y_{n_{k}}\right)<\frac{\varepsilon}{3 \cdot 2^{k-1}} .
$$

Notice that $\left(y_{n_{k}}\right)$ is f-Cauchy because, for every $m>k$, one has using (3.19)

$$
\begin{aligned}
\mathrm{d}\left(y_{n_{k}}, y_{n_{m}}\right) & \leq \mathrm{d}\left(y_{n_{k}}, y_{n_{k+1}}\right)+\mathrm{d}\left(y_{n_{k+1}}, y_{n_{k+2}}\right)+\cdots+\mathrm{d}\left(y_{n_{m-1}}, y_{n_{m}}\right) \\
& <\frac{\varepsilon}{3 \cdot 2^{k}}+\frac{\varepsilon}{3 \cdot 2^{k+1}}+\cdots+\frac{\varepsilon}{3 \cdot 2^{m-1}}<\frac{\varepsilon}{3 \cdot 2^{k-1}} .
\end{aligned}
$$

The space $(X, \mathrm{~d})$ being $\mathrm{f}$-complete, it follows that there exists $x \in X$ such that $y_{n_{k}} \stackrel{f}{\longrightarrow} x$. Now, since $y_{n_{k}} \in A_{n_{k}} \subset \overline{\bigcup_{n \geq k} A_{n}}$, one deduces that $x \in \overline{\bigcup_{n \geq k} A_{n}}$ for all $k \geq 1$, thus $x \in A$.

As, by hypothesis, $y_{n_{k}} \stackrel{b}{\longrightarrow} x$, we get $K \in \mathbb{N}$ such that $\mathrm{d}\left(y_{n_{k}}, x\right)<\frac{\varepsilon}{3}$ for all $k \geq K$. Consequently,

$$
\mathrm{d}(y, x) \leq \mathrm{d}\left(y_{n_{0}}, y_{n_{k}}\right)+\mathrm{d}\left(y_{n_{k}}, x\right)<\frac{\varepsilon}{3}+\frac{\varepsilon}{3 \cdot 2}+\cdots+\frac{\varepsilon}{3 \cdot 2^{k-1}}+\frac{\varepsilon}{3}<\varepsilon .
$$


From this, $y$ being arbitrarily chosen, we conclude that

$$
\mathrm{D}\left(A_{m}, A\right)=\sup _{y \in A_{m}} \inf _{x \in A} \mathrm{~d}(y, x) \leq \varepsilon
$$

Inequality (3.18) now follows from Step I and Step II. The proof is complete.

\section{Application. Iterated function systems}

In the following we adapt [26, Lemma 4.1] to our setting.

Lemma 4.1 Assume that in the quasi-metric space $(X, \mathrm{~d}) f$-convergence implies $b$-convergence, and let $F \in \mathcal{F}, \psi \in \Psi$ and $\omega: X \rightarrow X$ be a forward $\psi F$-contraction. Then the mapping $A \mapsto \omega(A)$ is a $\psi F$-contraction from $\left(\mathcal{K}_{\mathrm{f}}(X), h\right)$ into itself. If, in addition, $F$ is continuous, then the mapping $A \mapsto \overline{\omega(A)}$ is a $\psi F$-contraction from $\left(\mathcal{C}_{\mathrm{f}}(X), h\right)$ into itself.

Proof Choose $A, B \in \mathcal{K}_{\mathrm{f}}(X)$ such that $h(\omega(A), \omega(B))>0$. Assume that

$$
h(\omega(A), \omega(B))=\mathrm{D}(\omega(A), \omega(B))=\sup _{x \in A} \inf _{y \in B} \mathrm{~d}(\omega(x), \omega(y))>0 .
$$

By hypothesis,

$$
F(\mathrm{~d}(\omega(x), \omega(y))) \leq \psi(F(\mathrm{~d}(x, y))), \quad \forall x, y \in X, \omega(x) \neq \omega(y) .
$$

According to Proposition 2.4 and Remark 3.2, the mappings $\mathrm{d}$ and $\psi$ are continuous. So, since the set $A$ is compact, one can find $a \in A$ such that

$$
\mathrm{D}(\omega(A), \omega(B))=\inf _{y \in B} \mathrm{~d}(\omega(a), \omega(y))>0,
$$

hence $\mathrm{d}(\omega(a), \omega(y))>0$ for all $y \in B$. Therefore, for every $y \in B$,

$$
F(\mathrm{D}(\omega(A), \omega(B)))=F\left(\inf _{y \in B} \mathrm{~d}(\omega(a), \omega(y))\right) \leq F(\mathrm{~d}(\omega(a), \omega(y))) \leq \psi(F(\mathrm{~d}(a, y))),
$$

that is,

$$
F(h(\omega(A), \omega(B))) \leq \psi(F(\mathrm{~d}(a, y))), \quad \forall y \in B
$$

Let $b \in B$ be such that $\mathrm{d}(a, b)=\inf _{y \in B} \mathrm{~d}(a, y)$. Then, by (4.2) and since $F$ and $\psi$ are increasing, one obtains

$$
\begin{aligned}
F(h(\omega(A), \omega(B))) & \leq \psi(F(\mathrm{~d}(a, b)))=\psi\left(F\left(\inf _{y \in B} \mathrm{~d}(a, y)\right)\right) \\
& \leq \psi\left(F\left(\operatorname{supinf}_{x \in A} \mathrm{~d}(x, y)\right)\right)=\psi(F(\mathrm{D}(A, B)) \\
& \leq \psi(F(h(A, B))) .
\end{aligned}
$$

Consequently, $F(h(\omega(A), \omega(B))) \leq \psi(F(h(A, B)))$. 
For the second part of the statement, we first notice that from the definition of $\psi$ we have $\psi(t)<t$ for all $t \in(-\infty, \mu), \mu=\sup F$; hence $\psi(M)$ is b-bounded for every b-bounded set $B \subset X$. Next, assume that $A, B \in \mathcal{C}_{\mathrm{f}}(X)$ are such that $h(\overline{\omega(A)}, \overline{\omega(B)})=\mathrm{D}(\overline{\omega(A)}, \overline{\omega(B)})>0$. Then, by the continuity of $F, \psi$, and d, taking into account that $F$ and $\psi$ are increasing, we get, using Corollary 3.3(c),

$$
\begin{aligned}
F(h(\overline{\omega(A)}, \overline{\omega(B)})) & =F(h(\omega(A), \omega(B)))=F(\mathrm{D}(\omega(A), \omega(B))) \\
& =F\left(\sup _{x \in A} \inf _{y \in B} \mathrm{~d}(\omega(x), \omega(y))\right)=\sup _{x \in A} \inf _{y \in B} F(\mathrm{~d}(\omega(x), \omega(y))) \\
& \leq \sup _{x \in A} \inf _{y \in B} \psi(F(\mathrm{~d}(x, y)))=\psi\left(F\left(\sup _{x \in A} \inf _{y \in B} \mathrm{~d}(x, y)\right)\right) \\
& =\psi(F(\mathrm{D}(A, B))) \leq \psi(F(h(A, B))) .
\end{aligned}
$$

Definition 4.1 A family of self-mappings $\left(\omega_{k}\right)_{k=1}^{N}$ on a quasi-metric space $(X, \mathrm{~d})$ is called Iterated Function System (IFS for short). We say that the set map $\mathcal{S}: \mathcal{C}_{\mathrm{f}}(X) \rightarrow \mathcal{C}_{\mathrm{f}}(X)$ defined by $\mathcal{S}(B):=\bigcup_{k=1}^{N} \overline{\omega_{n}(B)}$ is the Hutchinson or the fractal operator associated with the respective IFS. If there exists a unique set $A \in \mathcal{C}_{\mathrm{f}}(X)$ such that $\mathcal{S}(A)=A$, then $A$ is called the attractor of the IFS.

Remark 4.1 If $\left(\omega_{k}\right)_{k=1}^{N}$ is an IFS consisting of $\psi F$-contractions on the Hausdorff quasimetric space $(X, \mathrm{~d})$ (in particular f-convergence implies b-convergence, see Proposition 2.1), then $\mathcal{S}(B)=\bigcup_{k=1}^{N} \omega_{k}(B)$ for every $B \in \mathcal{K}_{\mathrm{f}}(X)$. Indeed, $B$ being f-compact and $\omega_{k}$ continuous, it follows that $\omega_{k}(B)$ is $\mathrm{f}$-compact, hence $\mathrm{f}$-closed.

Theorem 4.1 Assume that in the quasi-metric space $(X, \mathrm{~d}) f$-convergence implies $b$ convergence and consider the IFS $\left(\omega_{k}\right)_{k=1}^{N}$, where, for each $k=1, \ldots, N, \omega_{k}: X \rightarrow X$ is a forward $\psi_{k} F$-contraction, $\psi_{k} \in \Psi, F \in \mathcal{F}$. If $F$ is continuous, then $\mathcal{S}$ is a $\psi F$-contraction in $\mathcal{C}_{\mathrm{f}}(X)$ for some $\psi \in \Psi$. If $(X, \mathrm{~d})$ is f-complete, then the IFS $\left(\omega_{k}\right)_{k=1}^{N}$ has a unique attractor $A \in \mathcal{C}_{\mathrm{f}}(X)$ which is approximated in the f-Hausdorff-Pompeiu metric by the sequence $\left(\mathcal{S}^{n}(B)\right)$ for every $B \in \mathcal{C}_{\mathrm{f}}(X)$.

Proof Set $\mu=\sup F$ and $\psi:(-\infty, \mu) \rightarrow \mathbb{R}, \psi=\max _{1 \leq k \leq N} \psi_{k}$. Clearly $\psi \in \Psi$.

Choose $A, B \in \mathcal{K}_{\mathrm{f}}(X)$ such that $h(\mathcal{S}(A), \mathcal{S}(B))>0$. By Corollary 3.3(d), we get

$$
0<h(\mathcal{S}(A), \mathcal{S}(B)) \leq \max _{1 \leq k \leq K} h\left(\overline{\omega_{k}(A)}, \overline{\omega_{k}(B)}\right)=h\left(\overline{\omega_{k_{0}}(A)}, \overline{\omega_{k_{0}}(B)}\right)
$$

for some $k_{0} \in\{1, \ldots, N\}$. Next, according to Lemma 4.1, one obtains

$$
\begin{aligned}
F(h(\mathcal{S}(A), \mathcal{S}(B))) & \leq F\left(h\left(\overline{\omega_{k_{0}}(A)}, \overline{\omega_{k_{0}}(B)}\right)\right) \leq \psi_{k_{0}}(F(h(A, B))) \\
& \leq \psi(F(h(A, B)))
\end{aligned}
$$

so $\mathcal{S}$ is a $\psi F$-contraction.

The last assertion follows from Theorem 3.4 and Corollary 3.2.

Theorem 4.2 We consider an IFS $\left(\omega_{k}\right)_{k=1}^{N}$ composed by forward $F_{k}$-contractions, $F_{k} \in \mathcal{F}$, $F_{k}$ continuous, $k=1, \ldots, N$, on the quasi-metric space $(X, \mathrm{~d})$ where $f$-convergence implies 
$b$-convergence. Then there is $F \in \mathcal{F}$ such that $\mathcal{S}$ is an F-contraction on $\mathcal{C}_{\mathrm{f}}(X)$. Furthermore, if the space is $f$-complete, then the IFS has a unique attractor in $\mathcal{C}_{\mathrm{f}}(X)$ approximated by $\left(\mathcal{S}^{n}(B)\right)$ for every $B \in \mathcal{C}_{\mathrm{f}}(X)$.

Proof By hypothesis, for each $k=1, \ldots, N$, there is $\tau_{k}>0$ such that

$$
\omega_{k}(x) \neq \omega_{k}(y) \quad \Rightarrow \quad \tau_{k}+F_{k}\left(\mathrm{~d}\left(\omega_{k}(x), \omega_{k}(y)\right)\right) \leq F_{k}(\mathrm{~d}(x, y)) .
$$

We set $F:=F_{1}+\cdots+F_{N}$ and $\tau:=\min _{1 \leq k \leq N} \tau_{k}$. Clearly $F \in \mathcal{F}$.

Let $A, B \in \mathcal{C}_{\mathrm{f}}(X)$ be such that $h(\mathcal{S}(A), \mathcal{S}(B))>0$. One can find $k_{0} \in\{1, \ldots, N\}$ such that

$$
0<h(\mathcal{S}(A), \mathcal{S}(B)) \leq \max _{1 \leq k \leq K} h\left(\overline{\omega_{k}(A)}, \overline{\omega_{k}(B)}\right)=h\left(\overline{\omega_{k_{0}}(A)}, \overline{\left.\omega_{k_{0}}(B)\right)} .\right.
$$

Now, using Lemma 4.1 and (3.3), we get

$$
\begin{aligned}
\tau+F(h(\mathcal{S}(A), \mathcal{S}(B))) & =\tau+\sum_{k=1}^{N} F_{k}(h(\mathcal{S}(A), \mathcal{S}(B))) \\
& \leq \tau_{k_{0}}+F_{k_{0}}\left(h\left(\overline{\omega_{k_{0}}(A)}, \overline{\left.\omega_{k_{0}}(B)\right)}\right)+\sum_{k \neq k_{0}} F_{k}\left(h\left(\overline{\omega_{k_{0}}(A)}, \overline{\omega_{k_{0}}(B)}\right)\right)\right. \\
& \leq F_{k_{0}}(h(A, B))+\sum_{k \neq k_{0}} F_{k}(h(A, B))=F(h(A, B)) .
\end{aligned}
$$

By Theorem 3.4 and Corollary 3.2 the rest of assertions follow.

Analogous theorems to Theorems 4.1 and 4.2 can be formulated by replacing the space $\mathcal{C}_{\mathrm{f}}(X)$ with $\mathcal{K}_{\mathrm{f}}(X)$, the proofs being similar.

Theorem 4.3 Let $(X, \mathrm{~d})$ be a quasi-metric space where f-convergence is equivalent to $b$ convergence and consider the IFS $\left(\omega_{k}\right)_{k=1}^{N}$ composed by forward $\psi_{k} F$-contractions, $\psi_{k} \in \Psi$, $F \in \mathcal{F}$. Then $\mathcal{S}$ is a $\psi F$-contraction in $\mathcal{K}_{\mathrm{f}}(X)$ for some $\psi \in \Psi$. If $(X, \mathrm{~d})$ is f-complete, then the IFS $\left(\omega_{k}\right)_{k=1}^{N}$ has a unique attractor in $\mathcal{K}_{\mathrm{f}}(X)$ which is approximated in the f-HausdorffPompeiu metric by the sequence $\left(\mathcal{S}^{n}(B)\right)$ for every $B \in \mathcal{K}_{\mathrm{f}}(X)$.

The following example is an application of each of Theorems 4.1 and 4.3.

Example 4.1 Let us consider $X=[1, \infty)$, the quasi-metric $\mathrm{d}: X \times X \rightarrow \mathbb{R}_{+} \cup\{0\}$ given by

$$
\mathrm{d}(x, y)= \begin{cases}\ln y-\ln x, & \text { if } y \geq x, \\ \frac{1}{3}(\ln x-\ln y), & \text { if } y<x\end{cases}
$$

and the mapping $F: \mathbb{R}_{+} \rightarrow \mathbb{R}, F(t)=\frac{1}{1-e^{t}}$. For $N \in \mathbb{N}$ and each $k=1,2, \ldots, N$, let us also define $\omega_{k}: X \rightarrow X$ and $\psi_{k}:(-\infty, 0) \rightarrow \mathbb{R}$ by

$$
\omega_{k}(x)=\frac{2 k x}{k x+1}, \quad \psi_{k}(t)=\sqrt[3]{t^{3}-1 / k^{3}}
$$


Then $\omega_{k}$ is a forward $\psi_{k} F$-contraction for all $k=1, \ldots, N$. Furthermore, the IFS $\left(\omega_{k}\right)_{k=1}^{N}$ has a unique attractor $A \in \mathcal{K}_{\mathrm{f}}(X) \subset \mathcal{C}_{\mathrm{f}}(X)$ which is approximated with respect to the $\mathrm{f}$ Hausdorff-Pompeiu metric by the sequence $(\mathcal{S}(B))$ for every $B \in \mathcal{C}_{\mathrm{f}}(X)$.

Proof It is easy to check the following properties: in the space $(X, \mathrm{~d}) \mathrm{f}$-convergence is equivalent to b-convergence, $\mathcal{K}_{\mathrm{f}}(X) \subset \mathcal{C}_{\mathrm{f}}(X), F \in \mathcal{F}, F$ is continuous, $\sup F=0$ and $\psi_{k} \in \Psi$ for every $k=1, \ldots, N$. In the same manner as in Example 3.1 we can prove that $(X, \mathrm{~d})$ is f-complete; hence, by Theorem 3.4 , the spaces $\left(\mathcal{K}_{\mathrm{f}}(X), h\right),\left(\mathcal{C}_{\mathrm{f}}(X), h\right)$ are complete.

In order to apply Theorem 4.1, respectively Theorem 4.3, it remains to prove that $\omega_{k}$ is a forward $\psi_{k} F$-contraction for each $k=1, \ldots, N$.

For this purpose, choose $k \in\{1, \ldots, N\}$ and $x, y \in X, x \neq y$. Notice that $\omega_{k}$ is an increasing map. Two cases can occur.

Case I: $x<y$. In this case

$$
\begin{aligned}
& \mathrm{d}(x, y)=\ln \frac{y}{x}, \quad \mathrm{~d}\left(\omega_{k}(x), \omega_{k}(y)\right)=\ln \frac{y(k x+1)}{x(k y+1)}, \quad F(\mathrm{~d}(x, y))=\frac{x}{x-y}, \\
& F\left(\mathrm{~d}\left(\omega_{k}(x), \omega_{k}(y)\right)\right)=\frac{x(k y+1)}{x-y}, \quad \psi_{k}(F(\mathrm{~d}(x, y)))=\frac{\sqrt[3]{k^{3} x^{3}-(x-y)^{3}}}{k(x-y)}
\end{aligned}
$$

Then

$$
\begin{gathered}
F\left(\mathrm{~d}\left(\omega_{k}(x), \omega_{k}(y)\right)\right) \leq \psi_{k}(F(\mathrm{~d}(x, y))) \quad \Leftrightarrow \quad \sqrt[3]{k^{3} x^{3}-(x-y)^{3}} \leq k x(k y+1) \\
\Leftrightarrow \quad \cdots \quad \Leftrightarrow \quad 0 \leq\left(k^{3}-1\right) y^{3}+3 x y(y-x)+x^{3}+3 k^{2} y^{2}+3 k y+1,
\end{gathered}
$$

where we omitted some elementary computations.

Case II: $x>y$. Then

$$
\begin{aligned}
& \mathrm{d}(x, y)=\ln \frac{\sqrt[3]{x}}{\sqrt[3]{y}}, \quad \mathrm{~d}\left(\omega_{k}(x), \omega_{k}(y)\right)=\ln \frac{\sqrt[3]{x(k y+1)}}{\sqrt[3]{y(k x+1)}}, \quad F(\mathrm{~d}(x, y))=\frac{\sqrt[3]{y}}{\sqrt[3]{y}-\sqrt[3]{x}}, \\
& F\left(\mathrm{~d}\left(\omega_{k}(x), \omega_{k}(y)\right)\right)=\frac{\sqrt[3]{y(k x+1)}}{\sqrt[3]{y(k x+1)}-\sqrt[3]{x(k y+1)}}, \\
& \psi_{k}(F(\mathrm{~d}(x, y)))=\frac{\sqrt[3]{k^{3} y-(\sqrt[3]{y}-\sqrt[3]{x})^{3}}}{k(\sqrt[3]{y}-\sqrt[3]{x})} .
\end{aligned}
$$

Hence

$$
\begin{aligned}
& F\left(\mathrm{~d}\left(\omega_{k}(x), \omega_{k}(y)\right)\right) \leq \psi_{k}(F(\mathrm{~d}(x, y))) \\
& \quad \Leftrightarrow \quad \frac{k^{3} y-(\sqrt[3]{y}-\sqrt[3]{x})^{3}}{k^{3}(\sqrt[3]{x}-\sqrt[3]{y})^{3}} \leq \frac{y(k x+1)}{(\sqrt[3]{x(k y+1)}-\sqrt[3]{y(k x+1)})^{3}} .
\end{aligned}
$$

The above inequality will result obviously from the following two relations:

$$
k^{3} y-(\sqrt[3]{y}-\sqrt[3]{x})^{3} \leq k^{3} y(k x+1)
$$


and

$$
\sqrt[3]{x(k y+1)}-\sqrt[3]{y(k x+1)} \leq \sqrt[3]{x}-\sqrt[3]{y}
$$

Indeed, inequality (4.3) is equivalent to

$$
0 \leq 3 \sqrt[3]{x y}(\sqrt[3]{x}-\sqrt[3]{y})+\left(k^{4} y-1\right) x+y
$$

which is obvious.

Now, we multiply inequality (4.4) by $\frac{1}{\sqrt[3]{x y}}$ and next we use the substitutions $a=\frac{1}{\sqrt[3]{x}}, b=$ $\frac{1}{\sqrt[3]{y}}$. Then $a<b$ and one has

$$
\sqrt[3]{k+b^{3}}-b \leq \sqrt[3]{k+a^{3}}-a
$$

which is clear because the mapping $t \mapsto \sqrt[3]{k+t^{3}}-t$ is decreasing.

Theorem 4.4 We consider an IFS $\left(\omega_{k}\right)_{k=1}^{N}$ composed by forward $F_{k}$-contractions, $F_{k} \in \mathcal{F}$, $k=1, \ldots, N$, on the quasi-metric space $(X, \mathrm{~d})$ where $f$-convergence is equivalent to $b$ convergence. Then there is $F \in \mathcal{F}$ such that $\mathcal{S}$ is an F-contraction on $\mathcal{K}_{\mathrm{f}}(X)$. Furthermore, if the space is f-complete, then the IFS has a unique attractor approximated by $\left(\mathcal{S}^{n}(B)\right)$ for every $B \in \mathcal{K}_{\mathrm{f}}(X)$.

Remark 4.2 The previous theorem improves [26, Th. 4.1] where the supplementary condition that the functions $g_{k}:=\max _{j} F_{j}-F_{k}$ must be increasing for all $k=1, \ldots, N$ is imposed.

\section{Acknowledgements}

The authors would like to thank the reviewers and the editors for their valuable suggestions and comments.

\section{Funding}

This work was supported by the project financed by Lucian Blaga University of Sibiu research grant LBUS-IRG-2018-04

Availability of data and materials

Not applicable.

Competing interests

The authors declare that they have no competing interests.

Authors' contributions

The three authors have equal share of contribution to the results. All three authors read and approved the final version of the manuscript.

\section{Author details}

${ }^{1}$ Department of Mathematics and Computer Science, Lucian Blaga University of Sibiu, Sibiu, Romania. ${ }^{2}$ Department of Mathematics, National Institute of Technology Calicut, Calicut, India. ${ }^{3}$ Department of Nonlinear Analysis, Faculty of Mathematics and Computer Science, University of Łódź, Łódź, Poland.

\section{Publisher's Note}

Springer Nature remains neutral with regard to jurisdictional claims in published maps and institutional affiliations.

Received: 8 December 2018 Accepted: 25 April 2019 Published online: 09 May 2019

\section{References}

1. Wilson, W.A.: On quasi-metric spaces. Am. J. Math. 53, 675-684 (1931)

2. Mennucci, A.: On asymmetric distances. Technical report, Scuola Normale Superiore, Pisa (2004)

3. Mainik, A., Mielke, A.: Existence results for energetic models for rate-independent systems. Calc. Var. Partial Differ. Equ. $22,73-99(2005)$ 
4. Mielke, A., Roubcek, T.: A rate-independent model for inelastic behavior of shape-memory alloys. Multiscale Model. Simul. 1, 571-597 (2003) (electronic)

5. Rieger, R.Z., Zimmer, J.: Young measure flow as a model for damage. Preprint 11/05, Bath Institute for Complex Systems, Bath, UK (2005)

6. Woon, W.L., Madnick, S.: Asymmetric information distances for automated taxonomy construction. Knowl. Inf. Syst. 21(1), 91-111 (2009)

7. Kelly, J.C.: Bitopological spaces. Proc. Lond. Math. Soc. s3-13(1), 71-89 (1963)

8. Aminpour, A.M.: Some results in asymmetric metric spaces. Math. Æterna 2(6), 533-540 (2012)

9. Brattka, V.: Generated quasi-metric hyper and function spaces. Topol. Appl. 127, 355-373 (2003)

10. Cobzaș, S.: Completeness in quasi-metric spaces and Ekeland variational principle. Topol. Appl. 158, 1073-1084 (2011)

11. Collins, J., Zimmer, J.: An asymmetric Arzelà-Ascoli theorem. Topol. Appl. 154, 2312-2322 (2007)

12. Gaba, Y.U.: Startpoints and $\alpha, \gamma$-contractions in quasi-pseudometric spaces. J. Math. 2014, Article ID 709253 (2014)

13. Khorshidvandpour, S., Mosaffa, M., Mousavi, S.M.: Some fixed point theorems in asymmetric metric spaces. Sci. Magna 9(2), 13-17 (2013)

14. Künzi, H.P.A.: A note on sequentially compact quasi-pseudo-metric spaces. Monatshefte Math. 95(3), 219-220 (1983)

15. Piri, H., Kumam, K.: Some fixed point theorems concerning F-contraction in complete metric spaces. Fixed Point Theory Appl. 2014, 210 (2014)

16. Reilly, I.L., Subrahmanyam, P.V., Vamanamurthy, M.K.: Cauchy sequences in quasi-pseudo-metric spaces. Monatshefte Math. 93, 127-140 (1982)

17. Wardowski, D.: Fixed points of a new type of contractive mappings in complete metric spaces. Fixed Point Theory Appl. 2012, 94 (2012)

18. Secelean, N.A., Wardowski, D.: \& F-Contractions: not necessarily nonexpansive Picard operators. Results Math. 70(3), 415-431 (2016)

19. Nazama, M., Arshada, M., Postolache, M.: Coincidence and common fixed point theorems for four mappings satisfying $(\alpha, F)$-contraction. Nonlinear Anal., Model. Control 23(5), 664-690 (2018)

20. Ali, M.U., Kamran, T., Postolache, M.: Solution of Volterra integral inclusion in b-metric spaces via new fixed point theorem. Nonlinear Anal., Model. Control 22(1), 17-30 (2017)

21. Kamran, T., Postolache, M., Ali, M.U., Kiran, Q.: Feng and Liu type F-contraction in b-metric spaces with application to integral equations. J. Math. Anal. 7(5), 18-27 (2016)

22. Secelean, N.A.: Weak F-contractions and some fixed point results. Bull. Iran. Math. Soc. 42(3), 779-798 (2016)

23. Hutchinson, J.: Fractals and self-similarity. Indiana Univ. Math. J. 30, 713-747 (1981)

24. Engelking, R.: General Topology. Heldermann, Berlin (1989)

25. Ćirić, L.B.: On contraction type mappings. Math. Balk. 1, 52-57 (1971)

26. Secelean, N.A.: Iterated function systems consisting of F-contractions. Fixed Point Theory Appl. 2013, 277 (2013)

27. Turinici, M.: Wardowski implicit contractions in metric spaces. arXiv:1212.3164v2 [Math.GN]

28. Barnsley, M.F.: Fractals Everywhere. Academic Press, San Diego (1988)

\section{Submit your manuscript to a SpringerOpen ${ }^{\circ}$ journal and benefit from:}

- Convenient online submission

- Rigorous peer review

- Open access: articles freely available online

- High visibility within the field

- Retaining the copyright to your article

Submit your next manuscript at $\boldsymbol{\nabla}$ springeropen.com 